Int. J. Dev. Biol. 58: 881-888 (2014)

doi: $10.1387 / \mathrm{ijdb} .150060$ at

\title{
The genome sequence of the corn snake (Pantherophis guttatus), a valuable resource for EvoDevo studies in squamates
}

\author{
ASIER ULLATE-AGOTE ${ }^{1,2,3}$, MICHEL C. MILINKOVITCH ${ }^{1,2,3}$ and ATHANASIA C. TZIKA ${ }^{1,2,3, *}$ \\ ${ }^{1}$ Laboratory of Artificial \& Natural Evolution (LANE), Dept. of Genetics \& Evolution, University of Geneva, ${ }^{2}$ SIB Swiss Institute \\ of Bioinformatics and ${ }^{3}$ Institute of Genetics and Genomics of Geneva (iGE3), University of Geneva, Geneva, Switzerland
}

\begin{abstract}
Squamates (snakes and lizards) exhibit a striking variety of phenotypes, with little known on their generative mechanisms. Studies aiming to understand the genetic basis of this wide diversity in morphology, physiology and ecology will greatly benefit from whole genome sequencing initiatives, as they provide the foundation for comparative analyses and improve our understanding of the evolution, development and diversification of traits. Here, we present the first draft genome of the corn snake Pantherophis guttatus, an oviparous snake that we promote as a particularly appropriate model species for evolutionary developmental studies in squamates. We sequenced 100-base paired-end reads from multiple individuals of a single family (parents and offspring) that produced a genome assembly of 1.53 gigabases (Gb), roughly covering $75 \%$ of the expected total genome size, and 297,768 scaffolds $>1 \mathrm{~Kb}$. We were able to fully retrieve 86 , and partially another 106, of the 248 CEGMA core genes, indicating that a high genome completeness was achieved, even though the assembly is fragmented. Using MAKER2, we annotated 10,917 genes with high confidence (Annotation Edit Distance $(A E D)<1$ ) and an additional 5,263 predicted genes matched with the species' transcriptome. Numerous colour and colour pattern morphs exist in $\boldsymbol{P}$. guttatus, making it an ideal model to study the genetic determinism, development, and evolution of adaptive colour traits in reptiles. Using our draft genome and a Single-Nucleotide Polymorphism (SNP) calling approach, we confirmed the interval with the causative mutation for the amelanistic phenotype, a result supported by a parallel exome-based study.
\end{abstract}

KEY WORDS: Pantherophis guttatus, corn snake, reptile, genome, amelanistic, SNP calling

\section{Introduction}

An increasing number of non-classical vertebrate genomes have been sequenced during the last decade, facilitating evolutionary and comparative developmental studies (Castoe et al., 2013, Vonk et al., 2013, Wang et al., 2013). However, the order Squamata is still largely underrepresented in these initiatives, despite the wide variety of phenotypes encountered within its 10,000 species (i.e., about twice as many as in mammals). In the last few years, the publications of the Anolis carolinensis genome (Alfoldi et al., 2011), the first draft (Castoe et al., 2011) and the improved version (Castoe et al., 2013) of the Burmese python (Python molurus) genome together with draft genomes of the king cobra Ophiophagus hannah (Vonk et al., 2013), of the rattlesnake Crotalus mitchellii (Gilbert et al., 2014) and of the common viper Vipera berus demonstrate an increased interest in Squamata in general, and snakes in particular,

\footnotetext{
Abbreviations used in this paper: AED, annotation edit distance; BLAST, basic local alignment search tool; CEGMA, core eukaryotic genes mapping approach; CR1, chicken repeat 1; DCT, dopachrome tautomerase; EST, expressed sequence tag; LINE, long interspersed nuclear elements; LTR, long terminal repeat; MIR, mammalian-wide interspersed repeats; Mya, millions years ago; OCA2, oculocutaneous albinism II; RTE, retro-transposable element; SINE, short interspersed nuclear element; SNP, single-nucleotide polymorphism; amel, amelanistic.
}

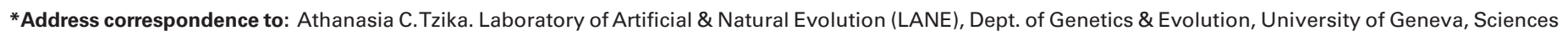

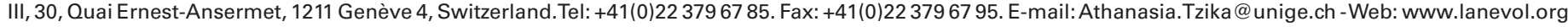

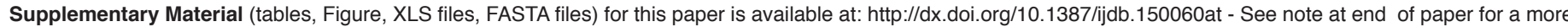
detailed description of the files.

Accepted: 9 February 2015.

ISSN: Online 1696-3547, Print 0214-6282

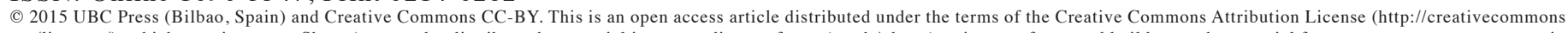

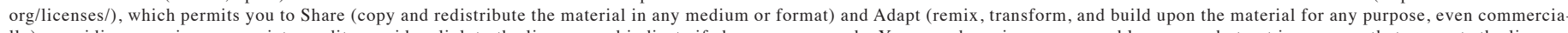

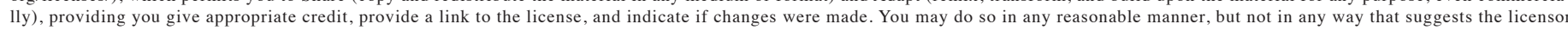
endorses you or your use. Printed in Spain 
as they are good models for investigating the mechanisms associated to their modified limb and body plan development (Di-Poi et al., 2010, Woltering, 2012), venom evolution (Vonk et al., 2013), physicochemical sensory perception (Brykczynska et al., 2013), extreme fluctuations in metabolic rates (Castoe et al., 2013), as well as development and patterning of scales and colours.

Over the last few years, we have been promoting the corn snake Pantherophis guttatus, of the Colubridae family and originating from North America, as a particularly appropriate snake model species for evolutionary developmental studies (Di-Poi et al., 2010, Milinkovitch and Tzika, 2007, Tzika and Milinkovitch, 2008). These animals are easy to breed and maintain, they have a moderate size (maximum length around 1.5 meters) and a long life span (>20 years), and are harmless to humans because they are nonvenomous and reluctant to bite. Of paramount importance is that corn snakes are oviparous (Fig. 1A), laying one to two clutches per year, making the species amenable to developmental studies (Fig. 1B). In addition, numerous colour and pattern morphs exist (Fig. 1C), making them ideal models to investigate the genetic determinism and underlying molecular pathways controlling adaptive colour variation in reptiles. In short, corn snakes combine a series of advantages matched by none of the other species of snakes for which a genome is available or in progress: Thamnophis species and rattlesnakes are ovoviviparous (hence, females must be sacrificed to access the embryos), whereas cobras, rattlesnakes and large pythons (such as Burmese pythons) are difficult or dangerous to maintain in captivity.

Here, we present the first draft genome of $P$. guttatus, produced by whole genome sequencing of multiple individuals from a single family (parents and offspring). The assembly statistics and the comparison to reference datasets show that this $P$. guttatus genome is of similar quality to other draft snake genomes (Castoe et al., 2011, Gilbert et al., 2014). In addition, we were able to use the assembled genomic sequence together with single-nucleotide polymorphism (SNPs) data to confirm the sequence interval containing the causal mutation of the amelanistic mutation (amel; lack of dark pigment from the skin and eyes, Fig. 2). Aggregation of SNPs identified a genomic area in $P$. guttatus that corresponds to a $31 \mathrm{Mb}$ region of A. carolinensis chromosome 3 and a $17 \mathrm{Mb}$ region of Gallus gallus chromosome 1. The present analysis confirms our previous work using an exome-assembly approach (Saenko et al., submitted) that identified a similar genomic interval and led to the identification of the causal ame/ mutation in corn snakes. Compared to the exome-based analysis, our genome-based approach provides longer genomic reference sequences that extend further into intronic and intergenic regions. Thus, more SNPs cosegregating with the amelanistic genotype are discovered, facilitating the retrieval of the interval of interest and increasing the number of SNPs that can be considered for posterior genotyping. Moreover, a shorter interval of $A$. carolinensis chromosome 3 was found with the genome-based $(31 \mathrm{Mb})$ than with the exome-based (39 Mb) approach.

\section{Results}

\section{The corn snake draft genome}

On five Illumina lanes (100-base paired-end reads), we sequenced genomic DNA from 78 individuals of a single corn snake family: (i) the mother, heterozygous for the recessive amelanistic mutation (amel/+), and the father, homozygous for the same mutation (amellame/) were indexed on a single lane, (ii) a pool of 20 non-indexed heterozygous amel/+ offspring, (iii) a pool of 20 non-indexed homozygous amelanistic offspring, and (iv and v) two pools, 18 non-indexed individuals each, of offspring grouped on the basis of another trait (unlinked to the amel locus). We used the filtered and trimmed Illumina reads (see Materials \& Methods) of the mother to assemble 26.1 giga-bases (Gb) into contigs. Our indirect estimate of the corn snake genome size (on the basis the k-mer distribution; see Materials and Methods) is 1.74-1.79 Gb (Supp. Fig. S1), a number in agreement with genome sizes of other Pantherophis and Elaphe species (1.8-2.2 Gb, Animal Genome Size Database www.genomesize.com). Hence, the draft genome presented here has an average sequencing depth of 14.6-15.0x. During this contig building step (i.e, assembling overlapping contiguous reads), we obtained 4,169,571 contigs $\geq 100 \mathrm{~b}$ with an average length of $358 \mathrm{~b}$ and an N50 of $563 \mathrm{~b}$ (Table 1). Afterwards, we ordered and oriented contigs into scaffolds, taking into account the paired-end reads information (i.e., the distance and orientation of the two reads of each sequenced pair). For the scaffolding, we used all six genomic libraries (184.6 Gb in total, 86-106-fold average genome sequencing depth). The genome assembly (deposited in GenBank, accession number JTLQ00000000, including only contigs/scaffolds $>200 \mathrm{~b}$ ) is $1.53 \mathrm{~Gb}$ long, i.e., between 70 and $85 \%$ of the expected genome size. It comprises 1,781,284 scaffolds and singletons $\geq 100 \mathrm{~b}$. The genome GC content is $39.81 \%$ and only $3.2 \%$ of the bases are unknown $(\mathrm{N})$. The great difference between the scaffold average length $(860 \mathrm{~b})$ and the $\mathrm{N} 50$ value $(3,674)$

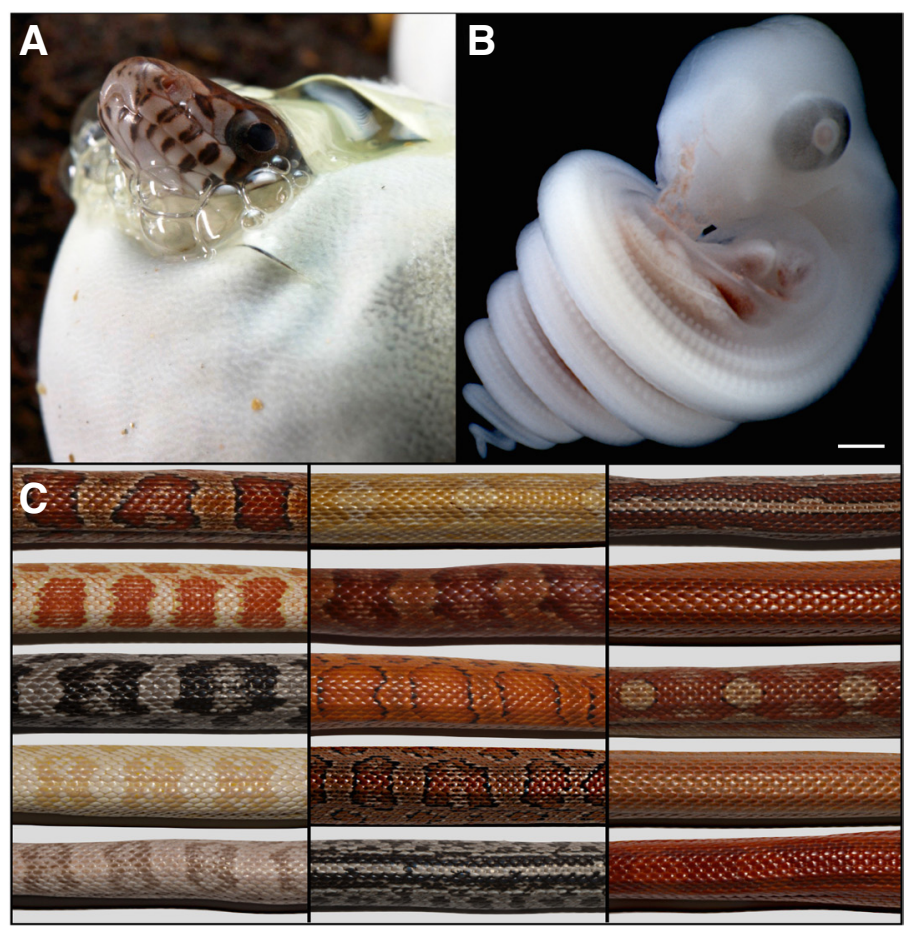

Fig. 1. The corn snake Pantherophis guttatus as a model species for evolutionary developmental studies. (A) Hatching after an egg incubation of 60 days. (B) Embryonic day 10 embryo. (C) Numerous colour and colour pattern morphs exist in Pantherophis guttatus, making it an ideal model to study the genetic determinism of adaptive colour traits in reptiles. 
TABLE 1

\section{CORN SNAKE GENOME ASSEMBLY STATISTICS}

\begin{tabular}{lcccccc} 
& $\begin{array}{c}\text { Sequences } \\
\geq 100 b\end{array}$ & $\begin{array}{c}\text { Assembly } \\
\text { size }(\boldsymbol{b})\end{array}$ & $\begin{array}{c}\text { Average } \\
\text { length }(\boldsymbol{b})\end{array}$ & $\mathbf{N 5 0}(\boldsymbol{b})$ & $\begin{array}{c}\text { Longest } \\
\text { sequence }(\boldsymbol{b})\end{array}$ & $\begin{array}{c}\text { Sequences } \\
\geq \mathbf{1 0 K b}\end{array}$ \\
\hline $\begin{array}{l}\text { Contig } \\
\text { assembly }\end{array}$ & $4,169,571$ & $1,493,759,741$ & 358 & 563 & 16,051 & 42 \\
Scaffolding & $1,781,284$ & $\begin{array}{c}1,532,089,567 \\
(3,2 \%)\end{array}$ & 860 & 3,674 & 102,731 & $\begin{array}{l}21,405 \\
(1.2 \%)\end{array}$ \\
\hline
\end{tabular}

In parenthesis, the percentage of unknown $(\mathrm{N})$ bases is indicated. $\boldsymbol{b}=$ number of bases

shows that many of the scaffolds are short: 297,768 scaffolds are $>1 \mathrm{~Kb}$ and half of the genome size is covered by 94,091 scaffolds (L50). These statistics are similar to those of the P. molurus and C. mitchellii draft genomes.

The Core Eukaryotic Genes Mapping Approach (CEGMA(Parra et al., 2007)), defines a set of 248 highly-conserved core proteins present in a wide range of eukaryotes (from yeast to human). Our assembly includes the complete gene sequence of 86 out of the 248 CEGMA eukaryotic core genes (34.68\%), a lower value compared to higher quality genomes (Castoe et al., 2013, Vonk et al., 2013), where more than 200 CEGMA full genes were sequenced. On the other hand, we were able to retrieve partial sequence of 192 core genes $(77.42 \%)$ from our draft genome, demonstrating that genome coverage is good but fragmented.

We built a library of $P$. guttatus repeats with RepeatModeler and combined it with all Vertebrate repeats from RepBase (Jurka et al., 2005). The use of this combined library resulted in masking $39.14 \%$ of the corn snake genomic sequence with RepeatMasker-open 4.0.5 (Smit et al., 1996). We observe a highly diverse landscape of repetitive elements (Fig. 3) as in other non-avian Sauropsida (Alfoldi et al., 2011, Shedlock et al., 2007) and unlike in humans, where most of the non-longterminal-repeats (nonLTR) retrotransposons consist of $L 1$ repeats. The most common long interspersed nuclear elements (LINEs) in the corn snake genome are homologous to the Chicken Repeat 1 (CR1, 2.81\%), with L1, L2, RTE (retro-transposable element) and R4 also being abundant, as in A. carolinensis (Novick et al., 2009). The identified

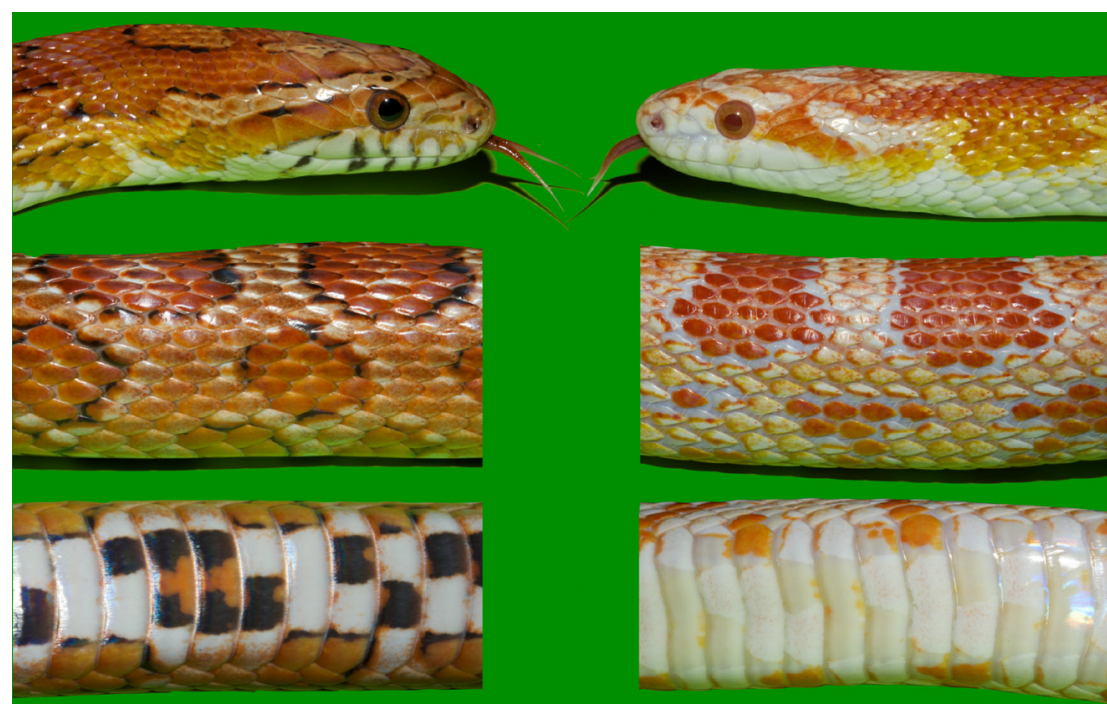

Fig. 2. Comparison of a wild-type (left) and an amelanistic (right) individual. The lack of melanin in the amelanistic individual is obvious in the eyes, around the dorso-lateral blotches, as well as by the absence of the ventral checker pattern. short interspersed nuclear elements (SINEs) mainly belong to the MIR-like family (1.91\%) and there are also a few LTR elements that are endogenous retroviruses. The distribution and variety of repetitive elements in the corn snake genome are similar to those of other snakes (Castoe et al., 2013), except that we observe a higher proportion of DNA transposons ("cut and paste DNA", $6.45 \%$ ). Finally, $16.58 \%$ of the corn snake genome corresponds to repetitive elements from unclassified groups (Fig. 3).

\section{Genome annotation}

Two iterations of the MAKER2 pipeline (Holt and Yandell, 2011) yielded 24,258 predicted genes. MAKER2 provides a set of gene predictors, performs similarity searches against cDNA/ESTs or proteins databases, and combines the outputs into a set of highquality gene predictions. Our corn snake transcriptomic data (Tzika et al., 2015) together with a protein database (including the SwissProt repository and NCBI sequences of $A$. carolinensis and snakes) were used in the MAKER2 pipeline to complement the gene models computed by three ab-initio/ evidence-based predictors: AUGUSTUS (Stanke and Waack, 2003), SNAP (Korf, 2004) and GeneMark-ES (Ter-Hovhannisyan et al., 2008). As a quality measurement of our annotation, we used the 'annotation edit distance' (AED, (Eilbeck et al., 2009)), which is based on the agreement at the nucleotide level (i.e., focusing on overlapping nucleotides rather than substitutions) between the final gene annotation and the aligned evidence data (cDNA/proteins) supporting that annotation. Values closer to zero correspond to higher quality annotation (i.e, it is more congruent with the cDNA or protein data) and predictions with an $A E D=1$ are interpreted as not supported by experimental data at all. An AED $<1$ was found for 10,917 of the 24,258 predicted genes (45\%) (Supp. Files S2-S4), with the majority of them showing an AED $<0.5(8,597,35.4 \%)$. These rather low numbers are due to the high fragmentation of the draft corn snake genome: many genes span multiple scaffolds, making their annotation impossible. Eighty percent $(8,817)$ of the corn snake proteins with AED $<1$ had a blastp hit (e-value $<10^{-5}$ ) against $A$. carolinensis (Ensembl v77 proteins), $P$. molurus or $O$. hannah sequences and $94 \%(10,237)$ had a megablast hit against our $P$. guttatus transcriptome (Tzika et al., 2015). When considering proteins with the maximum AED value of 1 , only $19.2 \%(2,565)$ had a hit against A. carolinensis, P. molurus or O. hannah and $39.4 \%$ $(5,263)$ against the $P$. guttatus transcriptome. The sequences without a hit against other species but with a hit against our corn-snake transcriptome are likely to be real cDNA/proteins, coded by less-conserved or taxonomically-restricted genes.

To further assess the completeness of the $P$. guttatus draft genome, we checked for the number of Hox genes annotated and/or present in the assembly (Suppl. Table S1). Eleven out of $40 \mathrm{Hox}$ genes were identified by MAKER2, with four being fully sequenced. We located homologous sequences of 27 additional Hox genes using BLAST searches on scaffolds $<10 \mathrm{Kbp}$ (not considered during the annotation process). The only missing Hox gene in our draft genome is Hoxd12, a gene involved in limb development in other vertebrates. This result is consistent with the absence of Hoxd12 in the fully- 
sequenced clusters of posterior Hox genes in $P$. guttatus (Di-Poi et al., 2010) and in the P. molurus, O. hannah and other squamata genomes (Vonk et al., 2013). Given the fragmented nature of the assembly, 29 of the 39 corn snake Hox genes were located on distinct scaffolds, besides five pairs of genes found on the same scaffold, preventing the confirmation of their cluster organisation.

\section{Identification of the genomic region with the amelanistic mutation}

We performed a Single-Nucleotide Polymorphism (SNP) calling approach to find the locus of the amelanistic (ame/) mutation. The Mendelian inheritance of this phenotype indicates that it is associated to a single recessive mutation in our captive-bred population. The homozygous amelanistic corn snake individuals can be identified by their lack of dark pigmentation (melanin) in their skin and eyes; hence, they miss both the checker pattern on the ventral skin and the black contour surrounding the red blotches on their dorsal side (Fig. 2).

We used the parental genomic DNA libraries (male amellamel and female amel/+) and the two libraries of their offspring pooled on the basis of their genotype (amellame/versus amell+). Each of the four libraries was aligned separately to our newly-assembled $P$. guttatus draft genome and the SNPs were extracted using FreeBayes (Garrison and Marth, 2012). We only kept biallelic SNPs, with a defined minimum sequencing depth, that were co-segregating according to the expected genotype for the amelanistic locus in each sample (see Supp. Table S2 and Materials and Methods). This filtering resulted in 19,104 SNPs distributed on 4,740 scaffolds (on average, 4 SNPs per scaffold and $1.16 \mathrm{SNPs} / \mathrm{Kb}$ ). Of these scaffolds, 751 had a megablast hit (bitscore $\geq 100$ ) against the $A$. carolinensis masked genome and included 5,273 SNPs (7 SNPs per sequence). Most of the hits (59\%) were against $A$. carolinensis Chromosome 3 , and 356 of them densely covered the $82-113 \mathrm{Mb}$ interval (Fig. 4A and 4B): 3,735 SNPs were present in this interval and only 131 elsewhere on the chromosome. Note that the SNPs in the $82-113 \mathrm{Mb}$ interval are split in two islands with only 50 hits found within the $87-97 \mathrm{Mb}$ sub-interval (corresponding, in $A$. carolinensis, to a gene desert, i.e., a region of the genome with very few protein-coding genes). As the $P$. guttatus and $A$. carolinensis lineages separated $\sim 166.4$ million years ago (Mya), it is not sur-

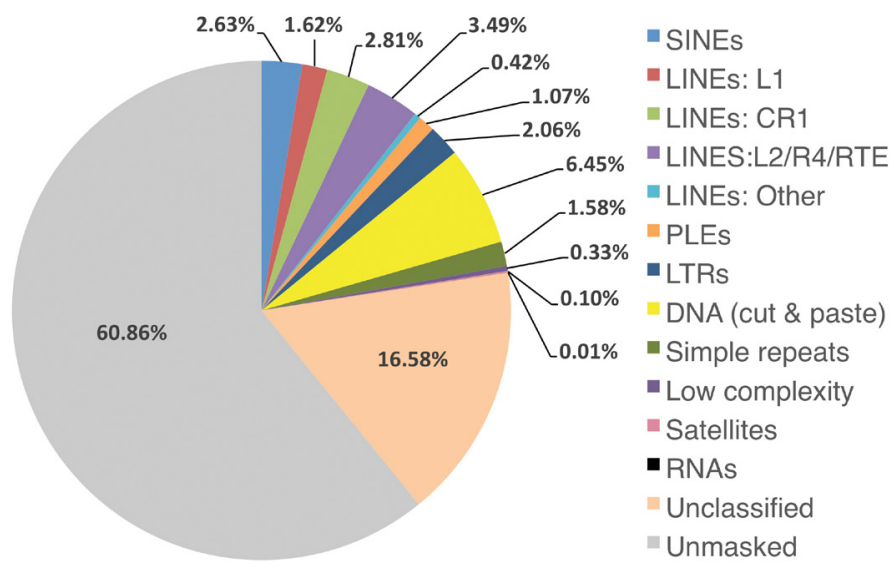

Fig. 3. Proportions of identified repetitive elements in the Pantherophis guttatus genome. prising that similarity searches (BLAST matches) are inefficient in this, probably fast evolving, intergenic region.

Another $61 P$. guttatus scaffolds with informative SNPs aligned to a 2.2 Mb A. carolinensis scaffold (GL343243.1), which includes genes orthologous to those located in the 132.9-134.4 Mb interval of the G. gallus chromosome 1 (Fig. 5). Other parts of the G. gallus chromosome 1 adjacent to this interval are syntenic with $A$. carolinensis chromosome 3 . Hence, our analysis indicates that the $A$. carolinensis scaffold GL343243.1 should probably be inserted in A. carolinensis chromosome 3 at about the position $109 \mathrm{Mb}$ (more specifically between the TGFBRAP1 and MGAT4A genes), i.e., in the area discussed above where most SNPs informative for the amelanistic locus are localised. Our analysis also indicates that genes present in the 131.8-132.9 Mb interval in G. gallus chromosome 1 are homologous to $A$. carolinensis genes located on three short scaffolds: GL343940.1 (0.1 Mb), GL343456.1 (0.66 Mb) and GL343542.1 (0.45 Mb). In total, 435 out of 751 (58\%) of the corn snake sequences with SNPs informative for the amelanistic locus had a hit against either one of these four $A$. carolinensis scaffolds or the 82-113 $\mathrm{Mb}$ interval of $A$. carolinensis chromosome 3. All these hits accounted for 4,674 SNPs (89\%) of the 5,273 informative SNPs (10.7 SNPs per scaffold and 1.45 SNPs/Kb), strongly suggesting that the amelanistic locus is in this interval, as SNPS located close to the amelanistic locus tend to present the same co-segregating pattern as the causal mutation.

We also ran a megablast search of the 4,740 filtered scaffolds against the G. gallus masked genome (International Chicken Genome Sequencing, 2004). As snakes are more distantly related to birds ( 274 Mya) than to lizards ( 166.4 Mya), only 291 $P$. guttatus scaffolds out of $4,740(6 \%)$ had a hit (bitscore $\geq 100)$. These matching scaffolds include 2,530 SNPs (13.2\%, 8.7 SNPs per scaffold, and 1.09 SNPs/Kb) and $226(77.5 \%)$ of them were against $G$. gallus chromosome 1, with 178 in the 129.5-146 Mb interval (2,312 SNPs with a density of 1.43 SNPs/Kb; Fig. 4C). These SNPs are distributed more uniformly than in $A$. carolinensis chromosome 3 (Fig. 4B), probably because this interval in the $G$. gallus chromosome 1 does not include a gene desert, contrary to the corresponding region of $A$. carolinensis chromosome 3 . The synteny between $G$. gallus chromosome 1 and the $A$. carolinensis chromosome 3 and the four short scaffolds (Fig. 5) provides additional support for the localisation of the amelanistic mutation.

A greater number of $P$. guttatus scaffolds had a megablast hit against the $O$. hannah and $P$. molurus genomes (than against the $A$. carolinensis and $G$. gallus genomes), as expected given their closer evolutionary relationship (Supp. Table S2). Although this larger number of $P$. guttatus scaffolds also corresponds to a greater number of informative SNPs, in total $85 O$. hannah and $120 P$. molurus scaffolds span the interval of interest on the $A$. carolinensis chromosome 3 , making the comparison with these snake species less informative in terms of synteny.

\section{Candidate genes for the amelanistic mutation}

Among the 205 genes in the $82-113 \mathrm{Mb}$ interval of the $A$. carolinensis chromosome 3 and the four short scaffolds (Fig. 5), two genes are particularly good candidates for bearing the mutation responsible for the amelanistic phenotype in corn snakes: the oculocutaneous albinism II or P protein (OCA2) at one end of the interval and the dopachrome tautomerase (DCT) or Tyrp2 genes at the other end (Fig. 4A). Both proteins participate in the 
A

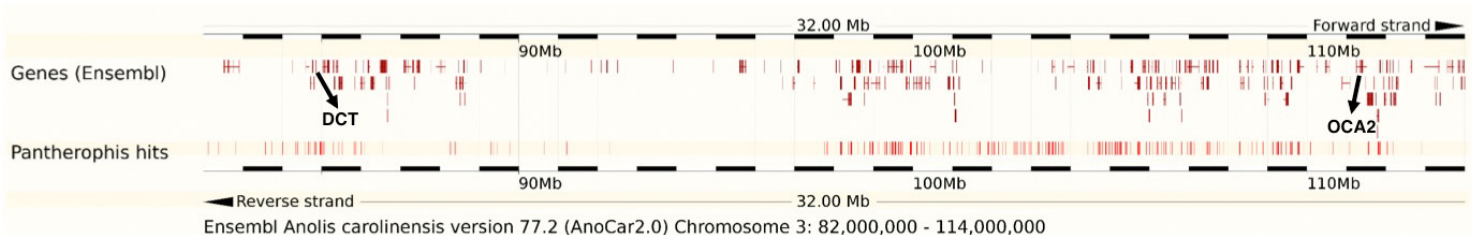

B
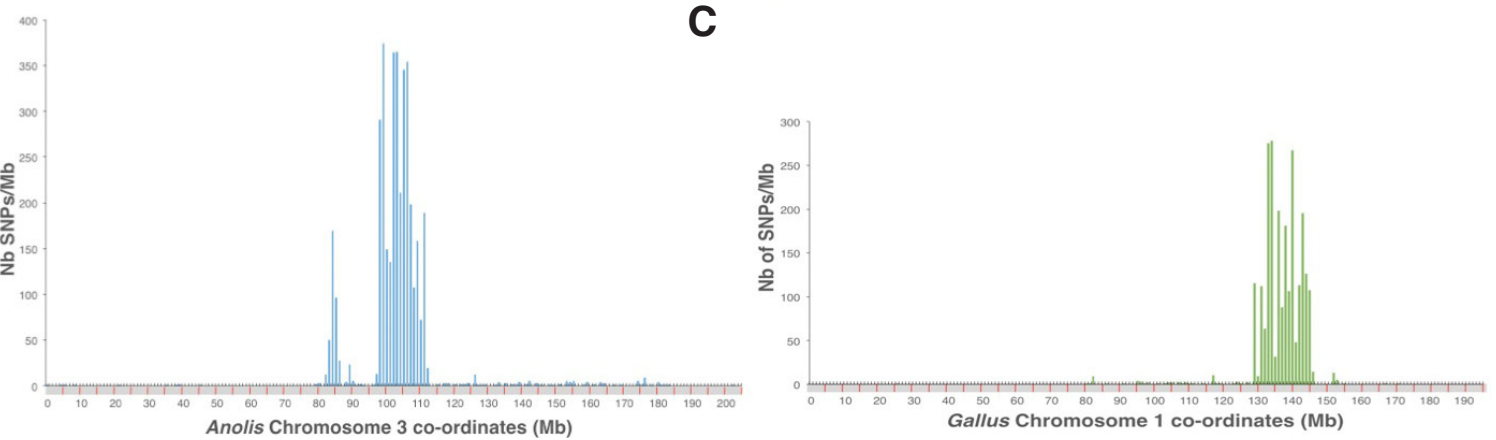

Fig. 4. Distribution of single-nucleotide polymorphisms (SNPs) and scaffolds on the interval harboring the amelanistic mutation. (A) Schematic representation of a $32 \mathrm{Mb}$ Anolis carolinensis chromosome 3 interval matching with Pantherophis guttatus sequences exhibiting SNPs co-segregating with the amelanistic mutation. Dark red bars at the top: Anolis carolinensis Ensembl genes. Light red bars at the bottom: location of the corn snake scaffolds BLAST hits. Black arrows: location of the two main candidate genes for the amel locus. (B,C) Distribution of Pantherophis guttatus SNPs that co-segregate with the amelanistic mutation and match (number of SNPs per Mb) with the Anolis carolinensis chromosome 3 (B) and the Gallus gallus chromosome 1 (C).

biosynthesis of eumelanin from tyrosine within the melanosomes of melanocytes. OCA2 is involved in the transport of the pigment out of the melanosomes, whilst DCT is an isomerase found at the organelle's membrane. Mutations in both genes are known to cause pigmentation disorders in humans and mice (Budd and Jackson, 1995, Rimoldi et al., 2014), resulting in lighter coloration of the hair, the skin and the eyes. These two genes were not annotated by the MAKER2 pipeline in our draft $P$. guttatus genome because their exons are distributed in several scaffolds.

Using a SNP calling analysis based on a $P$. guttatus exomeassembly approach (Saenko et al., submitted) rather than a genomeassembly (this study), we previously identified an overlapping, but longer, interval on $A$. carolinensis chromosome 3 as including the amelanistic locus. Even though the same sequencing data was used, the full-genome approach presented here allowed us to identify a higher number of co-segregating SNPs than with the exomeassembly approach. Indeed, the draft genome of the corn snake made it possible to extend the search for co-segregating SNPs further in the intronic and intergenic regions, and provided greater support for the interval containing the causal mutation. It is likely that the corn snake draft genome presented here will greatly assist the identification of mutations responsible for coloration phenotypes (Fig. 1C) or other traits in P. guttatus or closely-related species.

\section{Discussion}

Here, we have extended the scarce Squamata sequencing resources by assembling the first draft genome of the corn snake $P$. guttatus. We have long supported the use of this species as a model for evolutionary developmental biology in snakes (Brykczynska et al., 2013, Di-Poi et al., 2010, Milinkovitch and Tzika, 2007, Tzika et al., 2011, Tzika and Milinkovitch, 2008, Tzika et al., 2015). We are particularly interested in understanding the genetic basis of colour and colour pattern variation within and among snake species. The amelanistic mutation is the oldest mutation segregated in captive breeding populations. Since then, numerous other colour phenotypes (some of them due to single locus mutations) in corn snakes have been selected by breeders all over the world. Hence,

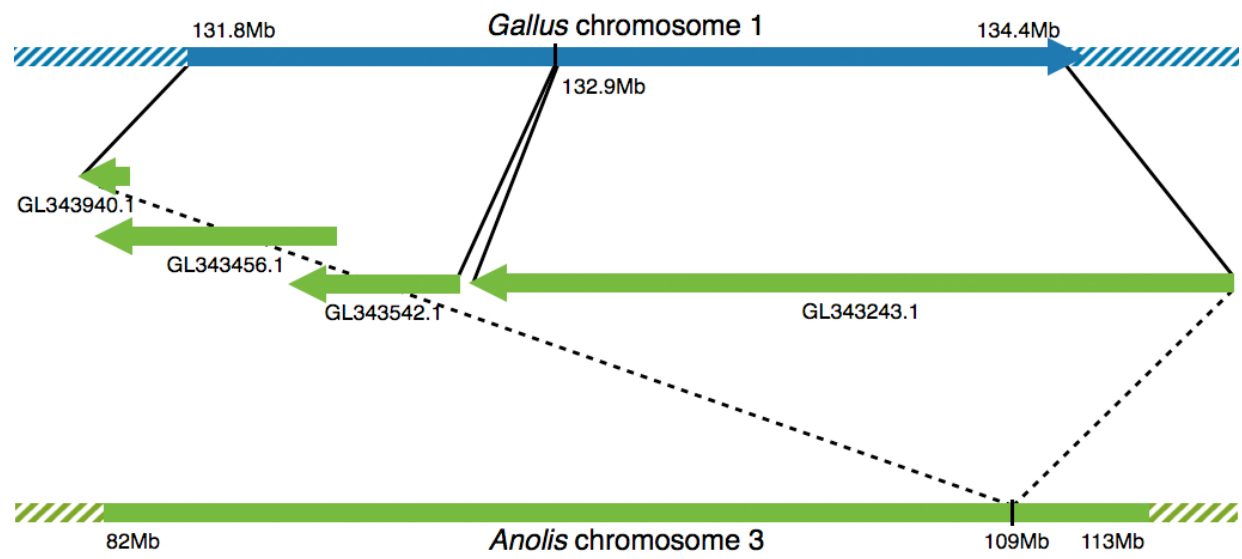

Fig. 5. Synteny diagram between the Anolis carolinensis (green) and the Gallus gallus (blue) genomic regions that match with most of the Pantherophis guttatus scaffolds harboring the single-nucleotide polymorphisms (SNPs) that co-segregate with the amelanistic mutation. Boxes with diagonal lines represent synteny between the two species independently of their SNP content. 
$P$. guttatus is a very good model to study the pigment biosynthesis pathways and developmental mechanisms controlling skin colour patterns in squamates.

The draft corn snake genome presented here shows similar quality and sequencing depth as other draft snake genomes, but is still rather fragmented. This high fragmentation explains that only 86 out of the 248 CEGMA highly-conserved core eukaryotic genes are completely sequenced, though at least 192 were partially retrieved. Nonetheless, we did obtain a core of 10,917 genes with a good prediction support and another 5,263 with transcriptomic support. So as to reduce the fragmentation of the corn snake genome sequence, it would be necessary to sequence pairedend Illumina libraries with variable fragment size and mate-paired Illumina libraries with inserts ranging from $3 \mathrm{~kb}$ to $6 \mathrm{~kb}$ to $40 \mathrm{~kb}$ (Nagarajan and Pop, 2013). Furthermore, generating sequence data with additional technologies, such as Pacific Biosciences, is likely to improve the assembly as recently put forward by the Assemblathon 2 initiative (Bradnam et al., 2013). Such an approach would produce longer scaffolds with less gaps and possibly resolve repetitive regions. Additional reads from a single corn snake, rather than a pool of several individuals (initially used for gene mapping; Saenko et al., submitted), would also increase the sequencing depth and accuracy of the final assembly.

We demonstrated the usefulness of the corn snake genomic sequence by retrieving the region in which the locus responsible for the amelanistic mutation (amel) is present. To that end, we performed a SNP calling approach using our genome assembly as a reference and Illumina libraries of individuals from a single $P$. guttatus family with known genotypes for the amel locus. Two excellent candidate genes were located in the selected interval, corresponding to $31 \mathrm{Mb}$ of the $A$. carolinensis chromosome 3 : DCT and OCA2. These two genes are involved in the melanin biosynthetic pathway and their mutations are known to produce pigmentation disorders in human and mice. Using our approach, we retrieved a greater number of informative SNPs on longer scaffolds, both in coding and intergenic/intronic regions, compared to the SNPS obtained in a parallel study, based on the same raw Illumina sequence data but using an exome-assembly approach (Saenko et al., submitted). In that study, we proceeded with the genotyping by PCR and Sanger sequencing of additional individuals of the same $P$. guttatus family and identified the mutation responsible for the corresponding phenotype.

\section{Materials and Methods}

\section{Whole-genome sequencing}

Genomic DNAwas extracted from tissue samples of $P$. guttatus individuals using the QIAGEN DNeasy Blood and Tissue kit. All animal experiments were performed in accordance with the Swiss animal welfare regulation (permit number 1008/3421/0). We obtained DNA from two adults: (i) an amelanistic male (i.e., homozygous for the recessive ame/mutation; amell amelgenotype), also heterozygous for the motleymutation (motley/+genotype) and (ii) a female heterozygous for the ame/mutation and homozygous for the motley mutation (amell+ and motley/motley genotype). Motley is a skin colour pattern morph that was not analysed in this study. In addition, we pooled DNA in equimolar concentrations in four offspring groups produced from these two individuals: (i) 20 heterozygous amel/+ individuals, (ii) 20 amelanistic (amellamel) animals, (iii) 18 snakes heterozygous for the motley mutation (motley/+) and (iv) 18 motley (motley/motley) individuals. We constructed DNA libraries (300-400bp fragment size) for each of the parents and for each of the offspring pools using the TruSeq PE Cluster Kit v3-cBot-HS. We then sequenced the samples on an Illumina HiSeq2000 sequencer, using one lane for each of the four libraries of pooled offspring and a fifth lane for the two indexed parental libraries.

We obtained 245 to 395 millions of Illumina 100-base paired-end reads per library and performed quality trimming using sickle v1.29 (Joshi and Fass, 2011); we removed flanking bases of quality $<20$ and we discarded reads that had one or more unknown base $(\mathrm{s})(\mathrm{N})$ in their sequence. We used SOAPec v2.01 (http:// soap.genomics.org.cn/soapdenovo.html) to remove the reads that were $<50 \mathrm{~b}$ and to further correct and filter the reads through the use of a k-mer distribution spectrum (for $k=23$ ) constructed using SOAPec. Reads with low-frequency $(\leq 3) \mathrm{k}$-mer regions are considered to contain sequencing errors and are selected by the program, which uses an algorithm to correct the bases producing the erroneous k-mers (if their quality is $<30$ ). The reads that remained uncorrected were then trimmed. Following the sequence clean-up, we obtained 21-37 Gb per library with paired-end reads (and single-end reads, when one of the reads from the pair is removed during the filtering process) showing an average length of $98 \mathrm{~b}$. We estimate the haploid genome size of $P$. guttatus to be $1.8-2.2 \mathrm{~Gb}$, based on the c-values of closely related Elaphe and Pantherophis species (Animal Genome Size Database -http:// www.genomesize.com) and the shared number of chromosomes between $P$. guttatus and $P$. obsoletus that practically makes their karyotype indistinguishable (Baker et al., 1971). Thus, we expect to have generated an average genome sequencing depth of $9.5 x-20.5 x$ per library.

We also indirectly estimated the corn-snake genome size based on the assumption that the genome sequencing depth is a function of the sequencing depth of the most frequent k-mers (Li et al., 2010). To this end, we obtained the frequency distribution of k-mers of different sizes $(17,23$ and 31-mers) in the raw sequencing reads of all the libraries using the program Jellyfish (Marcais and Kingsford, 2011). The peak k-mer depth was 92,84 and 74 , respectively (Supp. Fig. S1). Using the formula $M=$ $N(L-K+1) / L$, where $M$ is the k-mer peak depth, $N$ the genome sequencing depth, $L$ the average read length $(98.16 \mathrm{~b})$ and $K$ the selected k-mer length, we obtained a sequencing depth $N$ of 107-110x. Thus, we estimated the genome size at $1.74-1.79 \mathrm{~Gb}$ by dividing the total cumulated read length $(191,011,161,168 b)$ by the genome sequencing depth $N$. On the basis of this estimate, we expect to have generated an average genome sequencing depth of 14.6-15.0x.

\section{Genome assembly}

We assembled the $P$. guttatus genome using SOAPdenovo2-v2.04.240 (Luo et al., 2012). Only the 266 million filtered reads of the (amel/+) mother Illumina library were considered for the contig building step (i.e, assembling overlapping contiguous reads). We performed multiple assemblies with a range of k-mers between 43 and $55 \mathrm{~b}$ and selected the optimal k-mer length (45) on the basis of three assembly parameters:

(i) N50, (ii) N90 and (iii) the longest contig sequence. As k=43 and k=45 had similar statistics, we chose the longest k-mer. The average fragment size of each library was estimated using the software bwa v0.7.5a with default parameters by aligning a subset of its paired-end reads against the preliminary assembled contigs (Li and Durbin, 2009). For the scaffolding steps, the contigs were ordered and oriented using the paired-end reads information of all six Illumina libraries (parental and offspring). The scaffolding step was also performed in SOAPdenovo2 and all parameters were set to default, except for the $\mathrm{F}$ parameter that was activated to fill up gaps. Note that we performed assemblies with alternative approaches (e.g., by also using multiple libraries during contig building, in addition to scaffolding) and softwares, such as Platanus (Kajitani et al., 2014), but the quality of the final assembly did not improve.

The quality of the draft genome was assessed using CEGMAv2.5 (Parra et al., 2007), optimised for vertebrates. We also used RepeatModeler-1.0.8 to identify and model the $P$. guttatus repetitive elements. To mask the final assembly with RepeatMasker-open 4.0.5 (Smit et al., 1996), we considered 
the newly identified repeats together with all the Vertebrate repeats from RepBase Update 19.07 (Jurka et al., 2005).

\section{Genome annotation}

The Core Eukaryotic Genes Mapping Approach (CEGMA (Parra et al., 2007)) was used for quality control, but it additionally retrieves orthologs of the highly-conserved eukaryotic core genes in a genome, thus determining their exon-intron structure. We performed gene prediction using the automated pipeline MAKER2 (Holt and Yandell, 2011). We considered only those scaffolds that were longer than $10 \mathrm{~Kb}$ or that were $>1 \mathrm{~Kb}$ and had a predicted annotation using the CEGMA set of highly-conserved genes in eukaryotes. For the gene annotation, we built a protein database including the SwissProt database, as well as all $A$. carolinensis and snake proteins (including the ones from $P$. molurus and $O$. hannah) from NCBI. We also used a $P$. guttatus transcriptome assembled from Illumina and 454 cDNA libraries obtained from a mix of adult organs (testis, kidneys, brain and vomeronasal organ) and three developmental stages (Brykczynska et al., 2013, Tzika et al., 2015).

We run the first iteration of MAKER2 combining the evidence from known mRNAs and proteins and the ab-initio predictions of SNAP (Korf, 2004) and GeneMark-ES (Ter-Hovhannisyan et al., 2008). For this step, the SNAP hidden Markov models (HMM) were optimised using the CEGMA output and the GeneMark-ES model parameters were obtained from self-training in genome scaffolds greater than $10 \mathrm{~Kb}$. We then trained the evidencebased predictor AUGUSTUS (Stanke and Waack, 2003) with the output of the first step and we run it in a second MAKER2 iteration, together with the other two gene predictors. We also modeled new SNAP HMM from the output of the previous iteration. The repetitions library including the $P$. guttatus repeats identified by RepeatModeler and the Vertebrate repeats from RepBase Update 19.07 was used to mask the genome during the annotation process.

As an additional means to verify the genome completeness, we retrieved the Hox proteins of $A$. carolinensis from Ensembl version 77, of $P$. molurus from NCBI, and of the $O$. hannah scaffolds that include the Hox clusters. We then performed BLAST searches to find their homologous sequences in the $P$. guttatus draft genome.

\section{SNP calling}

To identify the genomic interval where the ame/mutation is located, SNP calling was performed on the genomic libraries of individuals (parents and offspring) with known genotype for the amel locus (excluding the libraries where offspring were segregated on the basis of the motley locus). First, using bwa v0.7.5a and default parameters, we aligned the reads of each library against the corn snake genomic scaffolds $>1 \mathrm{~Kb}$. Second, we converted the output to BAM files and extracted all variants using FreeBayes v0.9.9.2 (Garrison and Marth, 2012). We then used an inhouse Python script to extract from the output VCF file only those variants that (i) were biallelic SNPs (i.e., they had exactly two alleles), (ii) had a FreeBayes-estimated quality greater than 100 and (iii) had a sequencing depth between 8 and 50 for each library. At the next step, we filtered out the SNPs that deviated from the segregation expected for SNPs linked to the causal mutation. More specifically, considering only the two parental libraries, we discarded SNPs that met at least one of the following conditions: (i) presented both alleles in at least one amelanistic individual (i.e., homozygous for the ame/mutation) or (ii) showed one of the two alleles in less than $25 \%$ of the reads in the heterozygous parental library (amell+genotype) because these cannot be informative for mapping. Then, we used the four family libraries to perform the same filtering approach, but also discarding SNPs for which any of the two alleles was sequenced less than twice in the heterozygous offspring library. We compared the two filtered SNPs datasets to identify scaffolds that co-segregate with the amelanistic genotype, as they could hint to the location of the amelanistic mutation.

The identified scaffolds were compared against the $A$. carolinensis (AnoCar2.0, (Alfoldi et al., 2011)), G. gallus (Galgal4, (International Chicken Genome Sequencing, 2004)), O. hannah and P. molurus masked genomes using megablast (BLAST+ release 2.2.29). We considered only hits with an e-value $<10^{-5}$ and a bitscore $\geq 100$, keeping only the best match for each sequence.

\section{Supplementary Files}

Supplementary File S1 is a PDF including Supplementary Tables S1-S2 and Supplementary Fig S1;

Supplementary File $S 2$ is an XLS file with MAKER2 information on the annotated $P$. guttatus proteins $(A E D<1)$;

Supplementary Files S3 and S4are FASTA files with sequences of predicted proteins and transcripts $(A E D<1)$;

Supplementary File S5 is an XLS file listing the SNPs selected for mapping the amelanistic mutation.

\section{Acknowledgments}

We are grateful to Adrien Debry and Rosa Afonso for maintenance of the P. guttatus colony and Suzanne Saenko for setting up the crosses and extracting the DNA. Mostcomputations were performed at the Vital-ITCentre for high-performance computing (www. vital-it.ch) of the SIB Swiss Institute of Bioinformatics. This work was supported by grants from the University of Geneva (Switzerland), the Swiss National Science Foundation (FNSNF, grant31003A_125060), and the SystemsX.ch initiative (projectEpiPhysX).

\section{References}

ALFOLDI, J., DI PALMA, F., GRABHERR, M., WILLIAMS, C., KONG, L., MAUCELI, E., RUSSELL, P., LOWE, C.B., GLOR, R.E., JAFFE, J.D. et al., (2011). The genome of the green anole lizard and a comparative analysis with birds and mammals. Nature 477: 587-591.

BAKER, R.J., BULL, J.J. and MENGDEN, G.A. (1971). Chromosomes of ElapheSubocularis (Reptilla-Serpentes), with Description of an in-Vivo Technique for Preparation of Snake Chromosomes. Experientia 27: 1228-1229.

BRADNAM, K.R., FASS, J.N., ALEXANDROV, A., BARANAY, P., BECHNER, M. BIROL, I., BOISVERT, S., CHAPMAN, J.A., CHAPUIS, G., CHIKHI, R. et al., (2013). Assemblathon 2: evaluating de novo methods of genome assembly in three vertebrate species. Gigascience 2: 10.

BRYKCZYNSKA, U., TZIKA, A.C., RODRIGUEZ, I. and MILINKOVITCH, M.C. (2013). Contrasted evolution of the vomeronasal receptor repertoires in mammals and squamate reptiles. Genome Biol Evol 5: 389-401.

BUDD, P.S. and JACKSON, I.J. (1995). Structure of the mouse tyrosinase-related protein-2/ dopachrome tautomerase (Tyrp2/Dct) gene and sequence of two nove slaty alleles. Genomics 29: 35-43.

CASTOE, T.A., DE KONING, A.P., HALL, K.T., CARD, D.C., SCHIELD, D.R., FUJITA, M.K., RUGGIERO, R.P., DEGNER, J.F., DAZA, J.M., GU, W. et al., (2013). The Burmese python genome reveals the molecular basis for extreme adaptation in snakes. Proc Natl Acad Sci USA 110: 20645-20650.

CASTOE, T.A., DE KONING, J.A., HALL, K.T., YOKOYAMA, K.D., GU, W., SMITH, E.N., FESCHOTTE, C., UETZ, P., RAY, D.A., DOBRY, J. et al., (2011). Sequencing the genome of the Burmese python (Python molurus bivittatus) as a model for studying extreme adaptations in snakes. Genome Biol 12: 406.

DI-POI, N., MONTOYA-BURGOS, J.I., MILLER, H., POURQUIE, O., MILINKOVITCH, M.C. and DUBOULE, D. (2010). Changes in Hox genes' structure and function during the evolution of the squamate body plan. Nature 464: 99-103.

EILBECK, K., MOORE, B., HOLT, C. and YANDELL, M. (2009). Quantitative measures for the management and comparison of annotated genomes. Bmc Bioinformatics 10: 67.

GARRISON, E. and MARTH, G. (2012). Haplotype-based variant detection from short-read sequencing. arXiv preprint arXiv:1207.3907.

GILBERT, C., MEIK, J.M., DASHEVSKY, D., CARD, D.C., CASTOE, T.A. and SCHAACK, S. (2014). Endogenous hepadnaviruses, bornaviruses and circoviruses in snakes. Proceedings. Biological sciences / The Royal Society 281: 20141122.

HOLT, C. and YANDELL, M. (2011). MAKER2: an annotation pipeline and genomedatabase management tool for second-generation genome projects. Bmc Bioinformatics 12: 491

INTERNATIONAL CHICKEN GENOME SEQUENCING, C. (2004). Sequence and 
comparative analysis of the chicken genome provide unique perspectives on vertebrate evolution. Nature 432: 695-716.

JOSHI, N.A. and FASS, J.N. (2011). Sickle: A sliding-window, adaptive, quality-based trimming tool for FastQ files (Version 1.33) [Software]. Available at https://github. com/najoshi/sickle

JURKA, J., KAPITONOV, V.V., PAVLICEK, A., KLONOWSKI, P., KOHANY, O. and WALICHIEWICZ, J. (2005). Repbase Update, a database of eukaryotic repetitive elements. Cytogen. Genome Res. 110: 462-467.

KAJITANI, R., TOSHIMOTO, K., NOGUCHI, H., TOYODA, A., OGURA, Y., OKUNO, M., YABANA, M., HARADA, M., NAGAYASU, E., MARUYAMA, H. et al., (2014). Efficient de novo assembly of highly heterozygous genomes from whole-genome shotgun short reads. Genome Res. 24: 1384-1395.

KORF, I. (2004). Gene finding in novel genomes. Bmc Bioinformatics 5: 59.

LI, H. and DURBIN, R. (2009). Fast and accurate short read alignment with BurrowsWheeler transform. Bioinformatics 25: 1754-1760.

LI, R.FAN, W.TIAN, G.ZHU, H.HE, L.CAI, J.HUANG, Q.CAI, Q.LI, B.BAI, Y. et al., (2010). The sequence and de novo assembly of the giant panda genome. Nature 463: 311-317.

LUO, R., LIU, B., XIE, Y., LI, Z., HUANG, W., YUAN, J., HE, G., CHEN, Y., PAN, Q. LIU, Y. et al., (2012). SOAPdenovo2: an empirically improved memory-efficient short-read de novo assembler. GigaScience 1: 18.

MARCAIS, G. and KINGSFORD, C. (2011). A fast, lock-free approach for efficient parallel counting of occurrences of k-mers. Bioinformatics 27: 764-770.

MILINKOVITCH, M.C. and TZIKA, A. (2007). Escaping the mouse trap: The selection of new Evo-Devo model species. In J. Exp. Zool. Part B: Molec. Dev. Evol., vol. 308, pp. 337-346.

NAGARAJAN, N. and POP, M. (2013). Sequence assembly demystified. Nat Rev Genet 14: 157-167.

NOVICK, P.A., BASTA, H., FLOUMANHAFT, M., MCCLURE, M.A. and BOISSINOT, S. (2009). The evolutionary dynamics of autonomous non-LTR retrotransposons in the lizard anolis carolinensis shows more similarity to fish than mammals. Molec. Biol. Evol. 26: 1811-1822.

PARRA, G., BRADNAM, K. and KORF, I. (2007). CEGMA: A pipeline to accurately annotate core genes in eukaryotic genomes. Bioinformatics 23: 1061-1067.
RIMOLDI, V., STRANIERO, L., ASSELTA, R., MAURI, L., MANFREDINI, E., PENCO S., GESU, G.P., DELLONGO, A., PIOZZI, E., SOLDÀ, G. et al., (2014). Functiona characterization of two novel splicing mutations in the OCA2 gene associated with oculocutaneous albinism type II. Gene 537: 79-84.

SHEDLOCK, A.M., BOTKA, C.W., ZHAO, S., SHETTY, J., ZHANG, T., LIU, J.S. DESCHAVANNE, P.J. and EDWARDS, S.V. (2007). Phylogenomics of nonavian reptiles and the structure of the ancestral amniote genome. Proc Natl Acad Sci USA 104: 2767-2772.

SMIT, A., HUBLEY, R. and GREEN, P. (1996). RepeatMasker Open-3.0. RepeatMasker Open-3.0. www.repeatmasker.org.

STANKE, M. and WAACK, S. (2003). Gene prediction with a hidden Markov mode and a new intron submodel. Bioinformatics 19 Suppl 2: ii215-ii225.

TER-HOVHANNISYAN, V., LOMSADZE, A., CHERNOFF, Y.O. and BORODOVSKY, M. (2008). Gene prediction in novel fungal genomes using an ab initio algorithm with unsupervised training. Genome Res. 18: 1979-1990.

TZIKA, A.C., HELAERS, R., SCHRAMM, G. and MILINKOVITCH, M.C. (2011) Reptiliantranscriptome v1.0, a glimpse in the brain transcriptome of five divergent Sauropsida lineages and the phylogenetic position of turtles. Evodevo 2: 19.

TZIKA, A.C. and MILINKOVITCH, M.C. (2008). A Pragmatic Approach for Selecting Evo-Devo Model Species in Amniotes. In Evolving Pathways: Key Themes in Evolutionary Developmental Biology, (ed. A, M. and G, F.). Cambridge University Press, pp.123-143

TZIKA, A.C., ULLATE-AGOTE, A., GRBIC, D. and MILINKOVITCH, M.C. (2015) Reptilian Transcriptomes v2.0: an extensive resource for Sauropsida genomics and transcriptomics. Genome Biol. Evol. In press (doi:10.1093/gbe/evv106)

VONK, F.J., CASEWELL, N.R., HENKEL, C.V., HEIMBERG, A.M., JANSEN, H.J., MCCLEARY, R.J.R., KERKKAMP, H.M.E., VOS, R.A., GUERREIRO, I., CALVETE, J.J. et al., (2013). The king cobra genome reveals dynamic gene evolution and adaptation in the snake venom system. Proc NatIAcad SciUSA 110:20651-20656.

WANG, Z., PASCUAL-ANAYA, J., ZADISSA, A., LI, W., NIIMURA, Y., HUANG, Z., LI C., WHITE, S., XIONG, Z., FANG, D. et al., (2013). The draft genomes of softshell turtle and green sea turtle yield insights into the development and evolution of the turtle-specific body plan. Nat Genet 45: 701-706.

WOLTERING, J.M. (2012). From Lizard to Snake; Behind the Evolution of an Extreme Body Plan. Curr. Genomics 13: 289-299. 


\section{Further Related Reading, published previously in the Int. J. Dev. Biol.}

Sexual dimorphism of AMH, DMRT1 and RSPO1 localization in the developing gonads of six anuran species

Rafal P. Piprek, Anna Pecio, Katarzyna Laskowska-Kaszub,Jacek Z. Kubiak and Jacek M. Szymura

Int. J. Dev. Biol. (2013) 57: 891-895

Dual embryonic origin of the hyobranchial apparatus in the Mexican axolotl (Ambystoma mexicanum)

Asya Davidian and Yegor Malashichev

Int. J. Dev. Biol. (2013) 57: 821-828

Clonal analyses in the anterior pre-placodal region: implications for the early lineage bias of placodal progenitors

Sujata Bhattacharyya and Marianne E. Bronner

Int. J. Dev. Biol. (2013) 57: 753-757

Amphibian interorder nuclear transfer embryos reveal conserved embryonic gene transcription, but deficient DNA replication or chromosome segregation

Patrick Narbonne and John B. Gurdon

Int. J. Dev. Biol. (2012) 56: 975-986

Origins of $\mathrm{Cdx} 1$ regulatory elements suggest roles in vertebrate evolution

Stephen J. Gaunt and Yu-Lee Paul

Int. J. Dev. Biol. (2011) 55: 93-98

Reptile scale paradigm: Evo-Devo, pattern formation and regeneration

Cheng Chang, Ping Wu, Ruth E. Baker, Philip K. Maini, Lorenzo Alibardi and Cheng-Ming Chuong

Int. J. Dev. Biol. (2009) 53: 813-826

Proteomics analysis of regenerating amphibian limbs: changes during the onset of regeneration

Michael W. King, Anton W. Neff and Anthony L. Mescher

Int. J. Dev. Biol. (2009) 53: 955-969

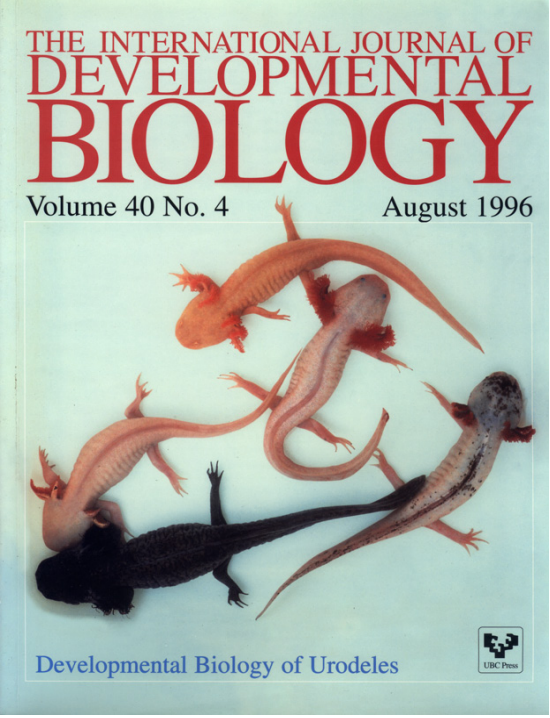

5 yr ISI Impact Factor $(2013)=\mathbf{2 . 8 7 9}$
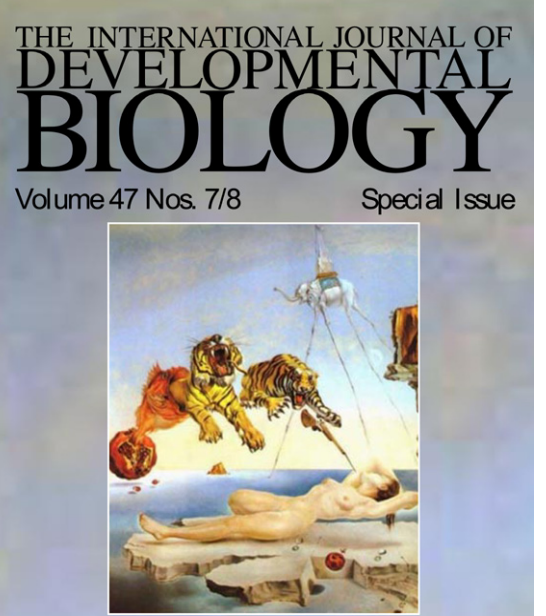

Evolution \& Development
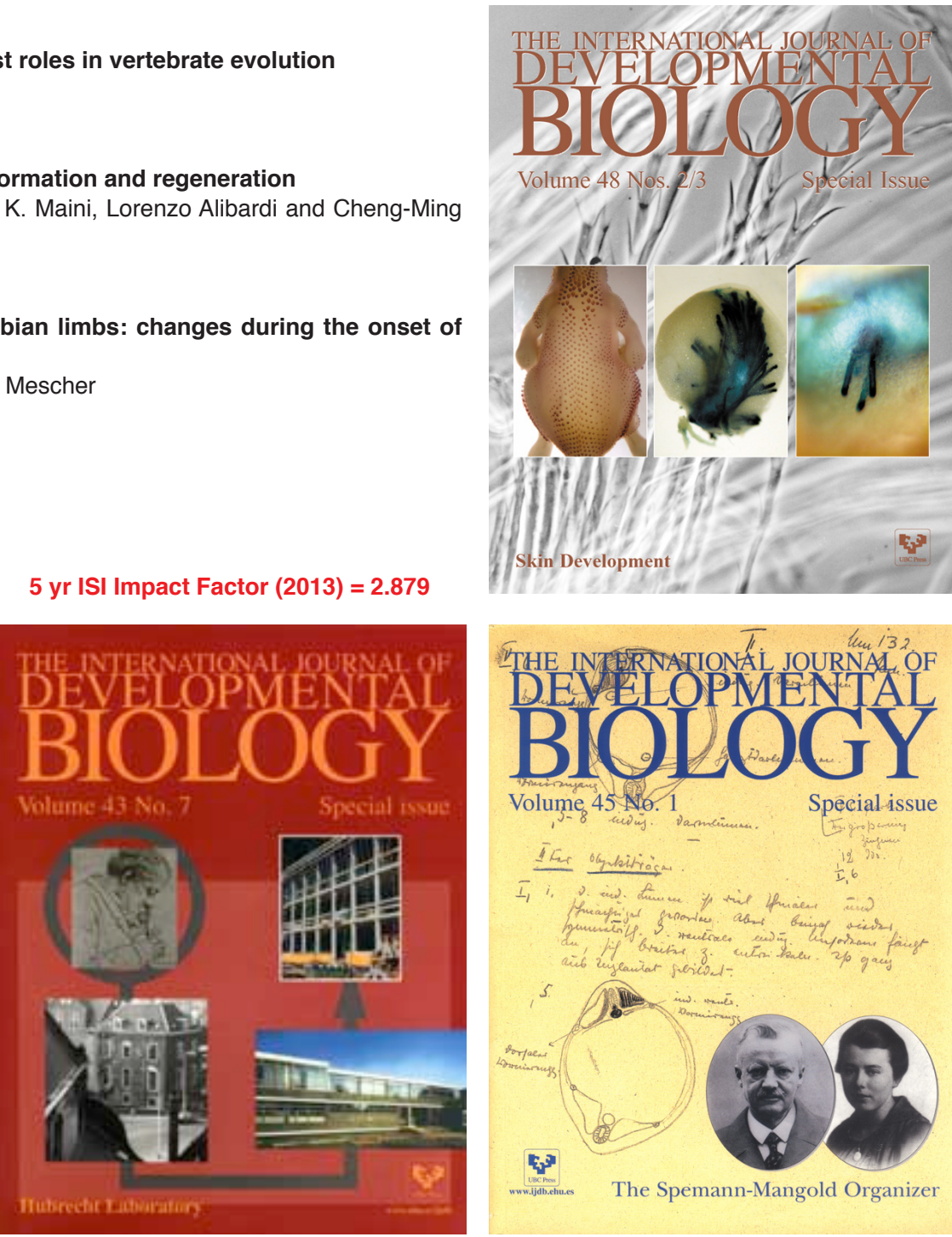

Volume 45 No. 1

Special-issue

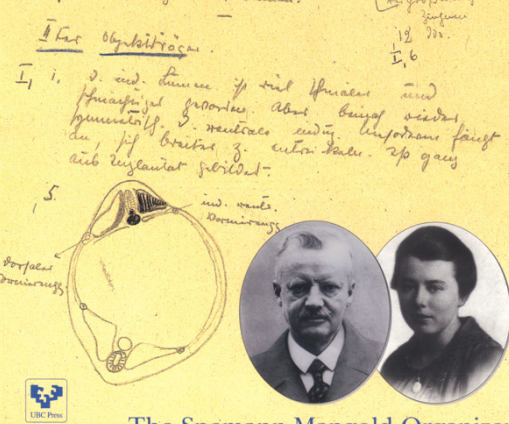

The Spemann-Mangold Organizer 